\title{
Characterization and analysis of azimuthally sensitive correlations
}

\author{
N Borghini \\ Service de Physique Théorique, CEA Saclay, F-91191 Gif-sur-Yvette cedex, \\ France
}

\begin{abstract}
A unified framework for describing the azimuthal dependence of two-particle correlations in heavy-ion collisions is introduced, together with the methods for measuring the corresponding observables. The generalization to azimuthal correlations between more than two particles is presented.
\end{abstract}

The high amount of data that modern nucleus-nucleus experiments can accumulate allow novel types of measurements, giving new information on the physics involved. An emerging class of experimental analyses thus consists in studying the dependence in azimuth of various observables in non-central collisions, investigating correlations with the impact-parameter direction.

A celebrated example of such a correlation with the reaction plane is the azimuthal dependence of single-particle emission, the so-called (one-particle) anisotropic flow (for a review, see [1]). In the following, we shall focus on the dependence with the azimuth of two-particle production. If the single-particle production is azimuthally dependent, the two-particle production depends on the azimuth as well. Beyond that, two arbitrary particles may also be correlated, for various reasons: a) they may be the decay products of a short-lived particle that decays before reaching the detectors, or b) they may both belong to a (di)jet originating from a hard parton scattering, or c) they may be identical particles whose wave-functions interfere. Whatever the physical mechanism involved, the resulting two-particle correlation will depend on the azimuths of the particles if the short-lived parent particles flow (case a), or because the anisotropy of the interacting region results in anisotropic patterns of parton energy loss [2] (case b) and interferometry [3] (case c).

The purpose of this paper is to present model-independent observables that characterize in a general way azimuthally-sensitive two-particle correlations, without any prejudice on the underlying physical mechanism [4]. We then discuss the experimental measurement of these observables, i.e., the measurement of the particlepair distribution with respect to the reaction plane. In particular, we shall mention methods of analysis that do not require to estimate the reaction plane, in opposition to those currently used [5-7]. Finally, we comment on the last step of the analysis, namely relating the observables we propose to models of the two-particle correlations. Quite obviously, that procedure is model-dependent, and we shall present several possible ways to tackle the issue. We shall conclude by generalizing the overall approach to correlations between more than two particles. 


\section{Two-particle anisotropic flow}

In this section, we shall introduce general observables for decribing the azimuthal dependence of two-particle correlations. A convenient approach consists in starting from the azimuthal dependence of one-particle emission, i.e., single-particle anisotropic flow; the generalization to two-body correlations then follows in a natural way.

Consider particles of a given type in a given rapidity $y$ and transverse momentum $p_{T}$ window. Denoting by $\Phi_{R}$ the orientation of the reaction plane (throughout the paper, azimuthal angles are measured with respect to a fixed direction in the laboratory), the probability distribution of the particle azimuth $\phi$ may be written as a Fourier series [8]:

$$
p\left(\phi-\Phi_{R}\right)=\frac{1}{2 \pi} \sum_{n=-\infty}^{+\infty} v_{n} \mathrm{e}^{\mathrm{i} n\left(\phi-\Phi_{R}\right)} .
$$

The azimuthal dependence of the particle distribution is thus entirely characterized by the coefficients $v_{n}=\left\langle\mathrm{e}^{-\mathrm{i} n\left(\phi-\Phi_{R}\right)}\right\rangle$, where angular brackets denote an average over particles and events. Note that the normalization choice implies $v_{0}=1$, while $v_{-n}=\left(v_{n}\right)^{*}$, where ${ }^{*}$ denotes the complex conjugate, since $p\left(\phi-\Phi_{R}\right)$ is real.

If the system is symmetric with respect to the reaction plane, equation (11) reads

$$
p\left(\phi-\Phi_{R}\right)=\frac{1}{2 \pi}\left[1+2 \sum_{n=1}^{+\infty} v_{n} \cos n\left(\phi-\Phi_{R}\right)\right]
$$

where $v_{n}=\left\langle\cos n\left(\phi-\Phi_{R}\right)\right\rangle$ is now a real number.

It is important to realize that characterizing anisotropic flow by the Fourier coefficients $v_{n}$ is a significant improvement with respect to older parameterizations. Thus, even though the azimuth of the impact parameter, $\Phi_{R}$, cannot be measured in each event, nevertheless the first $v_{n}$ can be reconstructed with accuracy, by performing a statistical analysis of the multiparticle azimuthal correlations between the detected particles (see section 2). The distribution $p\left(\phi-\Phi_{R}\right)$, on the other hand, cannot be measured accurately - this reflects the fact that for large $n$, the uncertainty on the extraction of $v_{n}$ becomes large [9]. In addition, since $v_{n}$ is an average quantity, it is easier to compute theoretically than the probability distribution for a definite azimuth with respect to the impact parameter, which varies considerably from event to event in computer simulations.

Keeping these advantages of Fourier coefficients in mind, we can now turn to azimuthally-sensitive two-particle correlations. This amounts to studying the distribution of particle pairs with respect to the reaction plane. Now, dropping for the sake of brevity rapidities and transverse momenta, a pair of particles with given types is described by two azimuthal angles $\phi_{1}$ and $\phi_{2}$. These can be combined into a "pair angle"

$$
\phi_{\text {pair }} \equiv x \phi_{1}+(1-x) \phi_{2},
$$

where $0 \leq x<1$, and the relative angle $\Delta \phi \equiv \phi_{2}-\phi_{1}$ (or any similar combination which does not depend on the orientation of the pair with respect to $\Phi_{R}$ ). Choosing the actual value of $x$ depends on the specific problem considered: a natural choice in interferometry and short-lived particle flow studies is that $\phi_{\text {pair }}$ represent the azimuth of the total transverse momentum $\mathbf{p}_{T 1}+\mathbf{p}_{T 2}$, while present studies of azimuthal correlations between high- $p_{T}$ particles rather adopt $x=1$, with the convention that particle 1 has the largest transverse momentum in the event (leading particle). In any 
case, the whole particle-pair orientation with respect to the reaction plane now affects only one azimuthal angle, namely $\phi_{\text {pair }}-\Phi_{R}$.

Once this change of angular variables has been performed, parameterizing the azimuthal dependence of two-particle correlations becomes straightforward. Consider a sample of pairs of particles with given types: the two-particle distribution depends on the two particle momenta and rapidities, and on the pair angle (with respect to the impact-parameter direction) and relative angle. Fixing $p_{T 1}, p_{T 2}, y_{1}, y_{2}$, and $\Delta \phi$ (in finite-width bins), the distribution is a function of $\phi_{\text {pair }}-\Phi_{R}$ only. By analogy with the single-particle anisotropic flow, equation (1), the probability distribution of the particle-pair azimuthal angle can be written as [4]:

$$
p\left(\phi_{\text {pair }}-\Phi_{R}\right)=\frac{1}{2 \pi} \sum_{n=-\infty}^{+\infty} v_{n}^{\text {pair }} \mathrm{e}^{\mathrm{i} n\left(\phi_{\text {pair }}-\Phi_{R}\right)} .
$$

The "pair-flow" Fourier coefficients are given by $v_{n}^{\text {pair }}=\left\langle\mathrm{e}^{-\mathrm{i} n\left(\phi_{\text {pair }}-\Phi_{R}\right)}\right\rangle$, where the average runs over particle pairs within the phase-space window and $\Delta \phi$ range selected; in particular, $v_{0}^{\text {pair }}=1$. The real-valuedness of the probability distribution implies $v_{-n}^{\text {pair }}=\left(v_{n}^{\text {pair }}\right)^{*}$, paralleling the similar property of the coefficients $v_{n}$.

However, unlike $v_{n}$, the pair-flow coefficient $v_{n}^{\text {pair }}$ is not a real number in general. This is due to the fact that the system is not symmetric under the change of $\phi_{\text {pair }}-\Phi_{R}$ into $-\left(\phi_{\text {pair }}-\Phi_{R}\right)$ while keeping $\Delta \phi$ constant. It follows that the real form of the Fourier expansion of $p\left(\phi_{\text {pair }}-\Phi_{R}\right)$ also contains sine terms, which could be omitted in equation (2):

$p\left(\phi_{\text {pair }}-\Phi_{R}\right)=\frac{1}{2 \pi}\left(1+2 \sum_{n=1}^{+\infty}\left[v_{c, n}^{\text {pair }} \cos n\left(\phi_{\text {pair }}-\Phi_{R}\right)+v_{s, n}^{\text {pair }} \sin n\left(\phi_{\text {pair }}-\Phi_{R}\right)\right]\right)$.

The real coefficients in expansion (5) are given by $v_{c, n}^{\text {pair }}=\left\langle\cos n\left(\phi_{\text {pair }}-\Phi_{R}\right)\right\rangle$, analogous to $v_{n}$, and $v_{s, n}^{\text {pair }}=\left\langle\sin n\left(\phi_{\text {pair }}-\Phi_{R}\right)\right\rangle$, which has no equivalent in the case of singleparticle flow. Quite naturally, the real and complex Fourier coefficients are related by $v_{n}^{\text {pair }}=v_{c, n}^{\text {pair }}-\mathrm{i} v_{s, n}^{\text {pair }}$. We shall further discuss the sine coefficients $v_{s, n}^{\text {pair }}$ later in section 3

\section{Analyzing two-particle flow}

We shall now focus on how to measure the pair-flow Fourier coefficients $v_{c, n}^{\text {pair }}, v_{s, n}^{\text {pair }}$. As in section 1 we first consider the case of the one-particle flow coefficients $v_{n}$, recalling existing methods of analysis. The methods for analyzing pair-flow coefficients will then emerge as straightforward generalizations.

Present analyses of anisotropic flow make use of various methods, which fall into three main categories. On the one hand, there are methods that rely on the determination of an estimate of the reaction plane [9-11]. By contrast, the other methods do not require such a step, but are based on a study of the azimuthal correlations between either two [12] or more-than-two particles [13,14]. Instead of characterizing methods according to whether or not they reconstruct the reaction plane, we shall see that another, perhaps more relevant way to classify them is to oppose two-particle-based methods to multiparticle ones.

The mostly used methods are those whose first step is to build event-by-event an estimate of the reaction plane, the so-called "event plane" [11]. Once this has been done and acceptance corrections have been performed to flatten its azimuthal 
distribution, one correlates the event-plane azimuth with that of each outgoing particle, thus obtaining the "differential flow" $v_{n}\left(p_{T}, y\right)$. This is a most natural idea: to measure distributions with respect to the impact-parameter direction, first determine the latter, then correlate outgoing particles with this direction. However, there are two caveats: first, the event plane does not coincide with the real reaction plane; this discrepancy is taken into account in actual analyses by correcting for the eventplane dispersion on a statistical basis [9]. The second, more serious issue, is the basic assumption of these methods, namely that all correlations between the event plane and a given particle, i.e., actually, all two-particle azimuthal correlations, are due to anisotropic flow only (or at least, that other sources of correlations are weak [10]). This assumption also underlies the computation of the event-plane dispersion through the help of "subevents" [9]. There clearly exist other sources of correlations between particles beyond flow: kinematic constraints between decay products, quantum interference between identical particles, inter- and intra-jet correlations... and these were shown to be of the same order of magnitude as the correlations due to flow at ultrarelativistic energies [15], which invalidates the assumption.

The second type of method is less intuitive than those reviewed above, but its principle is very simple [12]: assuming that all two-body azimuthal correlations are due to flow (and symmetry with respect to the reaction plane), the average $\left\langle\cos n\left(\phi_{2}-\phi_{1}\right)\right\rangle$ factorizes into $\left\langle\cos n\left(\phi_{1}-\Phi_{R}\right)\right\rangle\left\langle\cos n\left(\phi_{2}-\Phi_{R}\right)\right\rangle$, where $\phi_{1}$ and $\phi_{2}$ denote the azimuths of two particles from the same event. Averaging first over (pairs of) particles in the whole phase space covered by the detectors, one obtains $\left\langle\cos n\left(\phi_{2}-\phi_{1}\right)\right\rangle=\left(v_{n}\right)^{2}$, where the "integrated flow" $v_{n}$ is an average value of the corresponding Fourier coefficient. One can then restrict particle 2 to a small transverse momentum and rapidity window while letting particle 1 in the whole phase space, and average over all such possible pairs, which yields the differential flow through $\left\langle\cos n\left(\phi_{2}-\phi_{1}\right)\right\rangle=v_{n} v_{n}\left(p_{T 2}, y_{2}\right)$, where the first $v_{n}$ denotes the previously determined integrated flow. Now, to perform these averages in practice, one builds two-particle correlators similar to those used in interferometry studies, dividing a distribution of "real pairs" by a distribution of "background pairs" made by mixing particles from different events (which automatically removes acceptance effects) [12]. The ratio is then an even function of $\phi_{2}-\phi_{1}$ whose Fourier coefficients are precisely the two-particle averages $\left\langle\cos n\left(\phi_{2}-\phi_{1}\right)\right\rangle$ : by fitting the function, one can extract these averages, and thus the flow coefficients. Unfortunately, one realizes at once that the same problem of two-particle nonflow effects as above also plagues the analysis of flow through this method. $\ddagger$

Multiparticle methods of flow analysis were devised precisely to remedy the issue of nonflow correlations $[13,14]$. The idea of these methods is that when one considers cumulants of the correlations between an increasing number (four, six, eight) of particles, the relative magnitude of nonflow effects decreases (and, in practice, rapidly drops) while the magnitude of the correlations due to anisotropic flow grows, because flow is a collective behaviour, which affects all particles. One can even think of "infinite-order cumulants" that reflect collective effects only, isolating flow from other correlations: this is the purpose of the application of Lee-Yang zeroes to the analysis of flow [14]. By measuring cumulants or Lee-Yang zeroes of a properly chosen function, one can thus extract estimates of anisotropic flow that are unbiased by nonflow effects,

$\ddagger$ Note that the error on the flow estimates due to nonflow correlations is the same in both types of methods (using event planes or two-particle correlators), so that none of these methods is better than the others in that respect. 
i.e., with a smaller systematic error than two-particle methods. The price to pay is an increase in statistical uncertainties, but this increase is moderate in most cases, especially since cuts in phase space so as to diminish unwanted correlations are not necessary. As the two-particle correlation studies, multiparticle methods proceed in two successive steps, to obtain estimates first of integrated flow (using all detected particles in each event without any phase space restriction), then of differential flow.

We discussed methods for measuring single-particle anisotropic flow at length, for it turns out that only minor modifications of these methods are needed to measure the pair-flow coefficients $v_{c, n}^{\text {pair }}, v_{s, n}^{\text {pair }}$. More precisely, whatever the method, the first step is exactly the same: the determination of the event plane and its resolution in event-plane-based methods or the measurement of (single-particle) integrated flow in the methods based on two- or multiparticle correlations should be performed in the same way as in one-particle flow studies, without any change in the procedure.

The measurement of $v_{c, n}^{\text {pair }}$ is then strongly similar to that of differential flow: instead of correlating the azimuth $\psi$ of particles in a restricted phase-space bin to that of the event plane (event-plane methods) or of all other particles in the event (two- and multiparticle methods), one simply replaces $\psi$ by the pair angle $\phi_{\text {pair }}$. For instance, in event-plane methods the cosine coefficient is given by

$$
v_{c, n}^{\text {pair }}=\frac{\left\langle\cos n\left(\phi_{\text {pair }}-\Psi_{R}\right)\right\rangle}{\left\langle\cos n\left(\Psi_{R}-\Phi_{R}\right)\right\rangle},
$$

where $\Psi_{R}$ and $\Phi_{R}$ denote the azimuths of the event plane and of the reaction plane, respectively, while the average runs over pairs in the $\left(p_{T_{1}}, p_{T_{2}}, y_{1}, y_{2}, \Delta \phi\right)$ bin under study and over events.

Finally, the only significant change, which as we shall see amounts to replacing a $\cos$ by a sin, concerns the analysis of the sine coefficients $v_{s, n}^{\text {pair }}$. Within eventplane methods, $v_{s, n}^{\text {pair }}$ is given by the ratio of the average $\left\langle\sin n\left(\phi_{\text {pair }}-\Psi_{R}\right)\right\rangle$ over the resolution. With the two-particle-correlation method, the difference with singleparticle flow analyses is that the correlator is no longer an even function of the relative angle $\phi-\psi$ (where $\phi$ is any particle while $\psi$ is restricted to a "differential" bin). It is now a non-even function of $\phi-\phi_{\text {pair }}$ (where $\phi$ still is any particle in the event but those involved in the pair) whose cosine and sine Fourier coefficients are respectively

$$
\left\langle\cos n\left(\phi-\phi_{\text {pair }}\right)\right\rangle=v_{n} v_{c, n}^{\text {pair }} \quad \text { and } \quad\left\langle\sin n\left(\phi-\phi_{\text {pair }}\right)\right\rangle=v_{n} v_{s, n}^{\text {pair }},
$$

where $v_{n}$ denotes the single-particle integrated flow. In the multiparticle method of Lee-Yang zeroes, the necessary modification is once again minor: referring the reader to reference [14] for further detail on the implementation, $v_{s, n}^{\text {pair }}$ is given by equation (12) provided one replaces $\cos m n(\psi-\theta)$ in the numerator by $\sin m n\left(\phi_{\text {pair }}-\theta\right)$. If one wants to employ multiparticle cumulants, the change is slightly more important: to obtain $v_{s, n}^{\text {pair }}$, one must consider the imaginary parts (replacing $\psi$ by $\phi_{\text {pair }}$ ) of the cumulants defined by the power expansion of equations (26-27) in reference [13], instead of the real parts. All in all, however, whatever the method used, measuring pair flow does not represent a much greater difficulty than measuring single-particle flow, and ideally both measurements could be performed at once, since they share the same first step.

Although we emphasize that any method that can be used to measure singleparticle anisotropic flow $v_{n}$ can also be applied (modulo minor changes) to the measurement of two-particle flow, let us nevertheless make a further comment. As mentioned above, the various methods relying on two-particle correlations, including event-plane methods, assume that all correlations between two arbitrary particles in an 
event are caused by the correlation of each one to the reaction plane, i.e., one-particle anisotropic flow. If the purpose is to measure single-particle flow, the assumption introduces systematic errors in the determination of $v_{n}$. However, if the methods are employed so as to derive the pair-flow coefficients $v_{c, n}^{\text {pair }}, v_{s, n}^{\text {pair }}$, i.e., azimuthally dependent two-particle correlations, there is a logical inconsistency, since at some point it is supposed that such effects are negligible. Therefore, a logically coherent analysis should rather adopt multiparticle methods, despite the larger statistical uncertainty, making use of two-particle approaches to estimate systematic errors.

\section{Summary and applications}

In the preceding two sections, we have introduced a set of new observables, the pairflow Fourier coefficients $v_{c, n}^{\text {pair }}$ and $v_{s, n}^{\text {pair }}$, that characterize azimuthally-sensitive twoparticle correlations in a unified way, irrespective of the physics that generate the correlations. We then showed how to modify methods of single-particle flow analysis so as to measure these coefficients experimentally, again in a model-independent manner.

To gain some insight on the physics behind these two-particle correlations, a further step is needed, which we shall only sketch because it introduces some modeldependence which we deliberately want to avoid here. For this last step, two general approaches are possible. A first possibility is to start from already existing models of correlations, which make predictions for definite quantities, and to try to deal with the pair-flow observables so as to relate them to these quantities. This was attempted in reference [4], where we showed how to use the two-particle flow observables to recover previously-used quantities in models of quantum correlations [6] or high- $p_{T}$ jet-like correlations [7]. The second approach consists in predicting directly the values of the pair-flow coefficients $v_{c, n}^{\text {pair }}$ and $v_{s, n}^{\text {pair }}$ within the framework of given models, relating their behaviour to the various parameters of the models. An instance of such a prediction can be found in reference [4], where an identity relating together the various coefficients was derived in the case of correlations at large momentum between (jet) particles originating from high-energy partons that suffered in-medium energy loss.

Other similar predictions can be made on quite general grounds like symmetry properties. In particular, since the sine coefficients $v_{s, n}^{\text {pair }}$ are a novel feature that appears in two-particle anisotropic flow while being absent in single-particle flow, they are worth some further discussion in various physical situations.

Consider first the (one-particle) anisotropic flow of short-lived particles which rapidly decay into two daughter particles that reach the detector, e.g., $\rho \rightarrow \pi^{+} \pi^{-} . \S$ The $\rho$ mesons can only be reconstructed on a statistical basis, by correlating pion pairs, and extracting the $\rho$ flow can be done only by inspecting the azimuthal dependence of two-particle correlations between pions (sorted according to the pair invariant mass, rather than using the relative angle). In this specific case, if $\phi_{\text {pair }}$ is defined as the azimuth of the total transverse pair momentum, i.e., the parent $\rho$ transverse

$\S$ This can obviously also apply to particles that live longer, but still decay before they reach the detector, as $\Lambda \rightarrow p \pi^{-}$or $K_{S}^{0} \rightarrow \pi^{+} \pi^{-}$. For such particles, which are usually identified through their decay topology [5], at the expense of cuts which decrease the available statistics, one should correlate all pairs of possible daughters, without cuts. This results in an increased background (and smaller signal-to-background ratio) and the loss of event-by-event identification of the parent particles; but it also means larger statistics and thus smaller statistical errors on the final anisotropic flow values if the background is properly dealt with. 
momentum for pairs of daughter particles, then the sine coefficients $v_{s, n}^{\text {pair }}$ vanish when the colliding system is symmetric with respect to the reaction plane (the emission of $\rho$ mesons is then symmetric as well, unless parity is violated), while the cosine coefficients $v_{c, n}^{\text {pair }}$ coincide with the $\rho$-meson flow coefficients $v_{n}$.

In the case of short-range correlations between identical bosons due to the symmetrization of their pair wave-function, the symmetry between both particles implies that the sine coefficients will vanish, $v_{s, n}^{\text {pair }}=0$. Note, however, that Coulomb or strong-interaction correlations will spoil this identity, since they break the symmetry between both particles.

As a final word, let us briefly discuss the generalization to the dependence in azimuth of correlations between more than two particles. This may be of interest as a way to measure the anisotropic flow of particles that are identified in the detectors through at least three particles, as for instance $\Xi \rightarrow \Lambda \pi$ or $\Omega \rightarrow \Lambda K$ followed by $\Lambda \rightarrow p \pi^{-}[16]$, or $\omega \rightarrow \pi \pi \pi$. To tackle such a problem, the recipe is the same as for azimuthally-sensitive two-particle correlations: one should first perform a change of angular variables so as to isolate the dependence on the orientation with respect to the reaction plane in a single azimuth (a "triplet-angle") while the other variables are relative angles. Next, one only needs to consider particle triplets with fixed values of all these relative angles as well as of transverse momenta and rapidities, and write the three-particle probability distribution, which is now only a function of the tripletangle, as a Fourier series, similarly to equations (1) and (4). This defines a set of observables, the Fourier coefficients, a priori including both sine and cosine terms as in equation (5). Any method for analyzing pair anisotropic flow can then be applied to measure these three-particle flow coefficients, without modification (apart from the obvious replacement of $\phi_{\text {pair }}$ by the triplet angle). Finally, there enter modeldependent procedures to relate the observables to models.

\section{Acknowledgments}

I wish to thank the Hot Quarks 2004 organizers for their kind invitation and financial support. Stimulating discussions with Kirill Filimonov and Jean-Yves Ollitrault are gladfully acknowledged.

\section{References}

[1] Ollitrault J-Y 1998 Nucl. Phys. A 638 195c

[2] Wang X N 2001 Phys. Rev. C 63054902

[3] Tomašik B and Wiedemann U A 2004 Quark-Gluon Plasma 3 ed R C Hwa and X N Wang (Singapore: World Scientific) p 715 (Preprint hep-ph/0210250)

[4] Borghini N and Ollitrault J-Y 2004 Preprint nucl-th/0407041

[5] Adler C et al (STAR Collaboration) 2002 Phys. Rev. Lett. 89132301

[6] Heinz U W, Hummel A, Lisa M A and Wiedemann U A 2002 Phys. Rev. C 66044903

[7] Adams J et al (STAR Collaboration) 2004 Preprint nucl-ex/0407007

[8] Voloshin S and Zhang Y 1996 Z. Phys. C 70665

[9] Ollitrault J-Y 1997 Preprint nucl-ex/9711003

[10] Danielewicz P and Odyniec G 1985 Phys. Lett. B 157146

[11] Poskanzer A M and Voloshin S A 1998 Phys. Rev. C 581671

[12] Wang S et al. 1991 Phys. Rev. C 441091

[13] Borghini N, Dinh P M and Ollitrault J-Y 2001 Phys. Rev. C 64054901

[14] Bhalerao R S, Borghini N and Ollitrault J-Y 2003 Nucl. Phys. A 727373

[15] Borghini N, Dinh P M and Ollitrault J-Y 2000 Phys. Rev. C 62034902

[16] Castillo J (STAR Collaboration) 2004 J. Phys. G: Nucl. Part. Phys. 30 S1207 